\title{
Detection of an Intergalactic Meteor Particle with the 6-m Telescope
}

\author{
V.L. Afanasiev ${ }^{1}$, V.V. Kalenichenko ${ }^{2}$, and I.D. Karachentsev ${ }^{1}$ \\ 1 Special Astrophysical Observatory Russian Academy of Sciences, N. Arkhyz, KChR, 369167, Russia \\ 2 Astronomical Observatory, Kyiv Taras Shevchenko University, 3 Observatorna ul., Kyiv, 04053 Ukraine
}

Received: July 10, 2007/Revised: August 17, 2007

\begin{abstract}
On July 28, 2006 the 6-m telescope of the Special Astrophysical Observatory of the Russian Academy of Sciences recorded the spectrum of a faint meteor. We confidently identify the lines of FeI and MgI, OI, NI and molecular-nitrogen $\left(\mathrm{N}_{2}\right)$ bands. The entry velocity of the meteor body into the Earth's atmosphere estimated from radial velocity is equal to $300 \mathrm{~km} / \mathrm{s}$. The body was several tens of a millimeter in size, like chondrules in carbon chondrites. The radiant of the meteor trajectory coincides with the sky position of the apex of the motion of the Solar system toward the centroid of the Local Group of galaxies. Observations of faint sporadic meteors with FAVOR TV CCD camera confirmed the radiant at a higher than $96 \%$ confidence level. We conclude that this meteor particle is likely to be of extragalactic origin. The following important questions remain open: (1) How metal-rich dust particles came to be in the extragalactic space? (2) Why are the sizes of extragalactic particles larger by two orders of magnitude (and their masses greater by six orders of magnitude) than common interstellar dust grains in our Galaxy? (3) If extragalactic dust surrounds galaxies in the form of dust (or gas-and-dust) aureoles, can such formations now be observed using other observational techniques (IR observations aboard Spitzer satellite, etc.)? (4) If inhomogeneous extragalactic dust medium with the parameters mentioned above actually exists, does it show up in the form of irregularities on the cosmic microwave background (WMAP etc.)?
\end{abstract}

\section{INTRODUCTION}

Systematic searches for and investigation of meteors produced by the entry of cosmic bodies into the Earth's atmosphere are conducted with small wide-angle cameras 1. Slitless spectra yield spectroscopic data for meteors brighter than 4-5 magnitude and provide information on the chemical composition of the disintegrating particles 2]. An enormous number of sporadic meteors fainter than 8 magnitude are known to be observed besides sufficiently bright meteors associated with meteor streams. Observers on large telescopes often see very faint meteors in the fields of view of their instruments. In some cases, slit spectra of these meteors can be obtained 3 . In this paper we report the results of our analysis of the spectrum and radial velocity of a faint meteor that we accidentally detected in the field of view of the 6-m telescope of the Special Astrophysical Observatory of the Russian Academy of Sciences.

\section{OBSERVATIONS}

We recorded the spectrum of the meteor on July 28, 2006 during our observations of the spectra of faint galaxies in
Send offprint requests to: V.L. Afanasiev, e-mail: vafan@sao.ru

the $2206+29$ field using SCORPIO universal focal reducer attached to the 6-m telescope of the Special Astrophysical Observatory of the Russian Academy of Sciences and operating in multislit spectroscopy mode 4 . The spectrograph recorded the 3500-9500 $\AA$ wavelength interval with a spectral resolution of $15-18 \AA \AA$ via a $2048 \times 2048$ EEV42-40 CCD. We use standard observational technique with the spectrum referred to spectrophotometric standard GD248 at a similar zenith distance. We recorded the spectrum of the faint meteor simultaneously in three slits, thereby allowing the trajectory of the meteor on the sky to be confidently reconstructed. Figure 1 shows the sky area studied, slit locations, and the corresponding original images of the spectra. The dashed line shows the hypothetical meteor trajectory. The upper panel of Fig. 2 shows the images of the meteor spectra in three slits after the reduction of scale distortions, wavelength calibration, and sky-background subtraction. The lower panel shows the average spectrum of the meteor uncorrected for spectral sensitivity. The FeI and MgI lines typical of chondrites [5] can be confidently identified in the blue part of the spectrum, as also evidenced by the relative intensities of the FeI multiplets and MgI lines [6]. The red part of the spectrum features conspicuous OI and NI lines and molecular nitrogen $\left(\mathrm{N}_{2}\right)$ bands, which are typical of strongly heated 
center RA 220604.82 DEC + 2930 10.5, field of view $6 \times 3$ arcmin

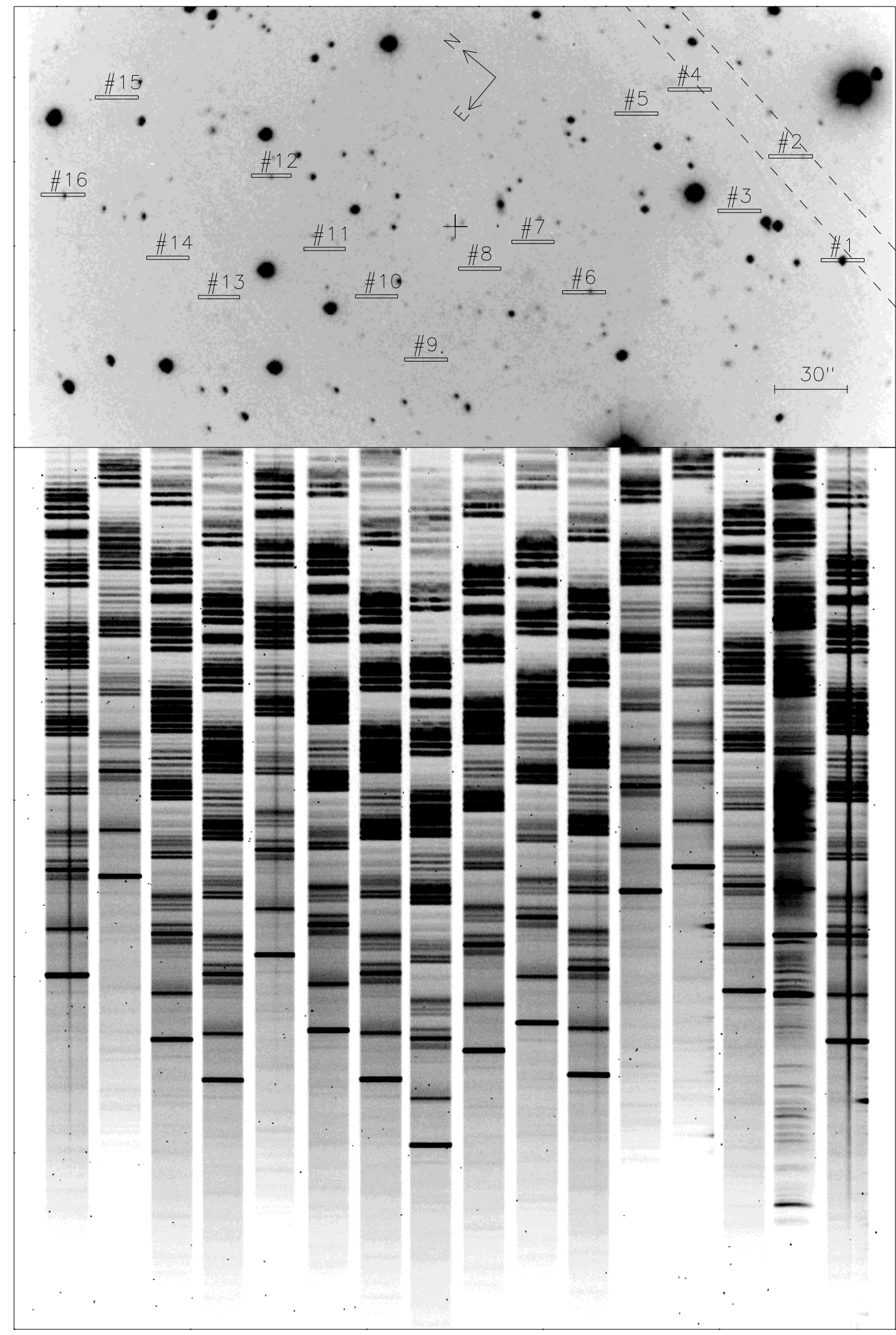

Fig. 1. Spectra in the field $2206+29$ taken with SCORPIO spectrograph operating in multislit mode and attached to the 6-m telescope of the Special Astrophysical Observatory of the Russian Academy of Sciences. The upper panel shows the region studied with the slit positions and numbers marked. The spectrum of the meteor was recorded in slits \#1,\#2, and \#4. The dashed line shows the hypothetical track of the meteor (its direction and width). 


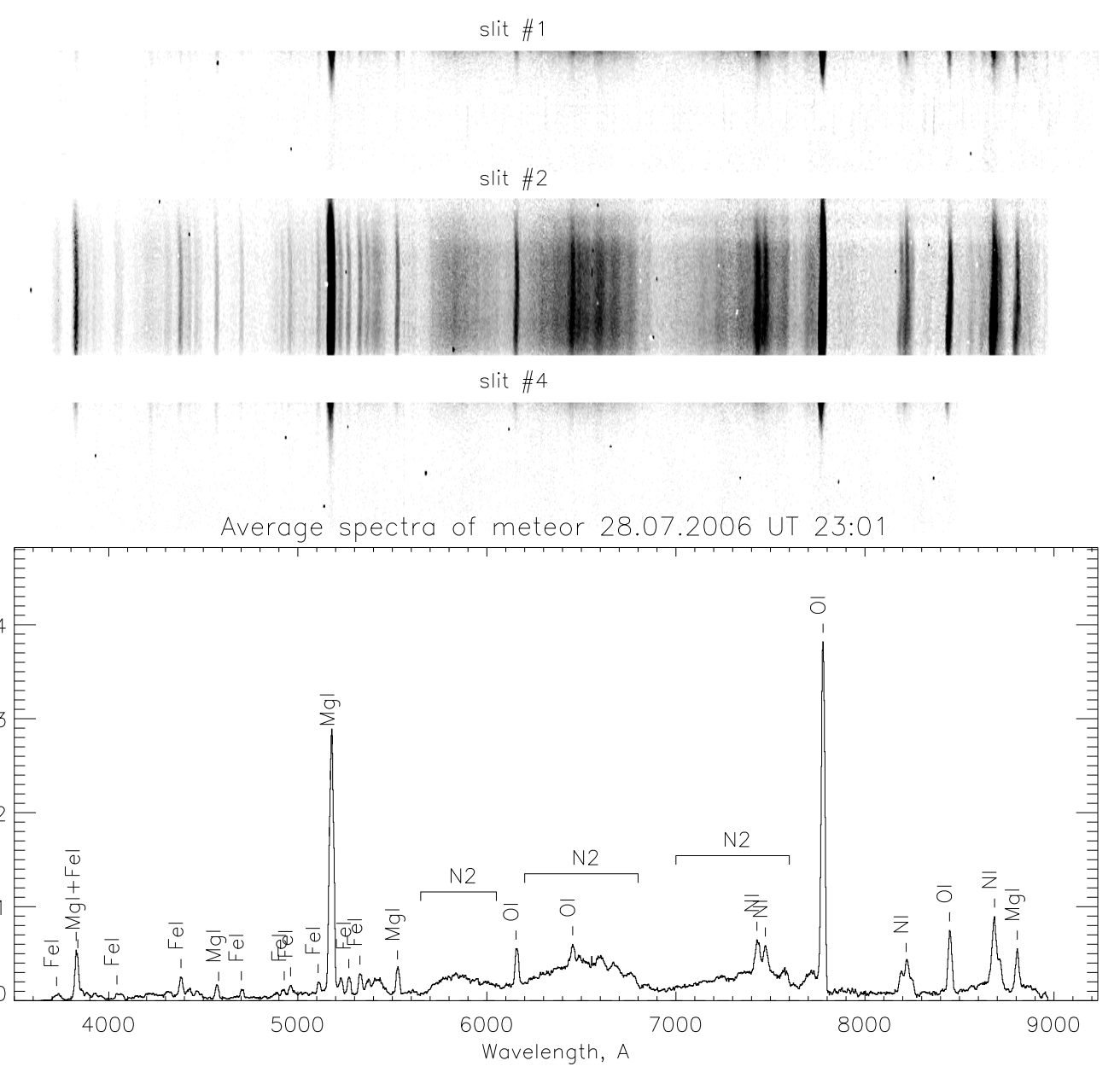

Fig. 2. The spectra of the meteor taken in slits \#1,\#2, and \#4 after subtraction of the sky background and wavelength calibration. The upper panel shows the images of the spectra in various slits and the lower panel shows the average spectrum of the meteor for slit \#2 with the lines identified.

air with the temperature of $15000-20000 \mathrm{~K}$ [7]. One can also see the infrared resonance $\mathrm{MgI}$ line, which belongs to the spectrum of the meteor particle proper.

The distortion of lines that shows up conspicuously on the spectra is most remarkable. It is indicative of the variation of radial velocity along the slit. The first estimates based on the original spectra as seen in the telescope yielded radial-velocity variations exceeding $250 \mathrm{~km} / \mathrm{s}$, which is absolutely unusual for meteors. To refine the radial-velocity variations, we determined the Doppler shifts by cross correlating the spectrum of the meteor along the slit with the average spectrum calibrated to the laboratory wavelength scale. The left-hand panel in Fig. 3 shows the isophotes of the observed radial-velocity distribution (LOSVD) and the right-hand panel, the Doppler velocities along the slit measured from the position of the maximum. It is absolutely evident that this pattern corresponds to the spectrum of the products of evaporation of a meteor particle in the atmosphere - we see the deceleration portion corresponding to minimum velocity at the center of the slit and the portions of maximum velocity corresponding to meteor vapor at the ends of the spec- trum. It follows from this that the velocity of intrusion of the observed meteor into the Earth's atmosphere was about $300 \mathrm{~km} / \mathrm{s}$.

The distribution of geocentric velocities of meteors is known to have two peaks located at about $30 \mathrm{~km} / \mathrm{s}$ and in the $60-80 \mathrm{~km} / \mathrm{s}$ interval 8 . The first peak is attributed to solar system particles, whereas the second peak is attributed to particles captured by the Sun and moving in hyperbolic orbits. Radar data are indicative of the excess of meteors in the domain of high velocities (about 1\% of meteors have velocities above $100 \mathrm{~km} / \mathrm{s}$ ), however, no meteor observations are known to yield velocities of several hundred $\mathrm{km} / \mathrm{s}$ [9. Hence the question: what do we see?

The closest velocity - the linear velocity of the rotation of the Sun about the Galactic center - is equal to about $220 \mathrm{~km} / \mathrm{s}$ and is insufficient to explain the meteor velocity we observed. An analysis of the motions of LocalGroup galaxies 10 shows that the Solar System moves at a velocity of $316 \pm 11 \mathrm{~km} / \mathrm{s}$ toward the mass center of the group in the direction of $l=93 \pm 2^{\circ}$ and $b=-4 \pm 1^{\circ}$. Figure 4 compares the position of the apex of the motion toward the centroid of the Local Group with the trajectory 

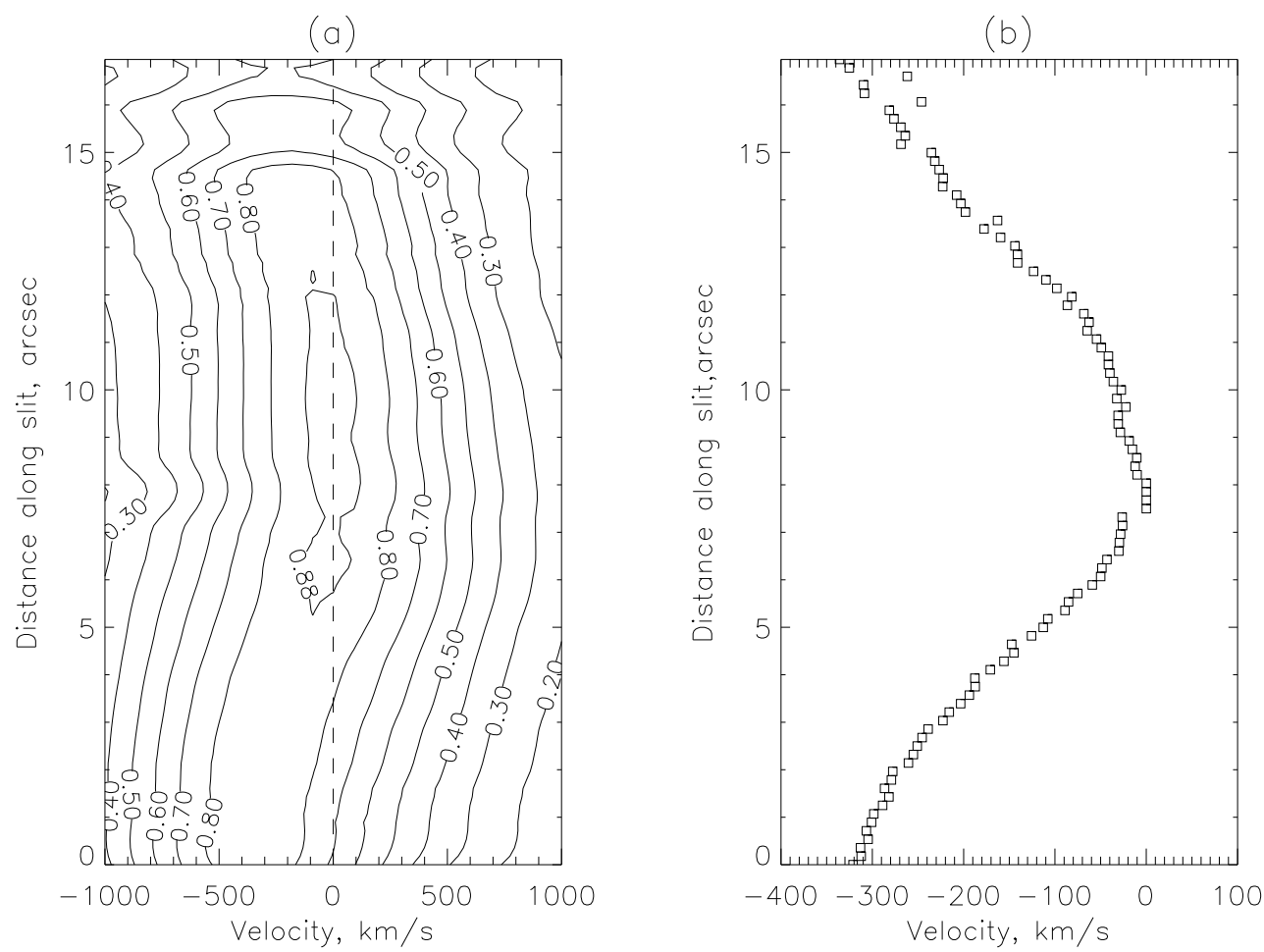

Fig. 3. Distortion of lines in the meteor spectrum taken using \#2. (a) Isophotes of the cross-correlation function computed for each point along the slit height and (b) the radial-velocity curve along the slit height.

of the meteor on the sky. As is evident from the figure, the agreement is remarkable: the radiant of the trajectory of our meteor coincides with the position of the apex. Given the agreement of velocities, this fact leads us to conclude that we observe an intergalactic particle, which is at rest with respect to the mass centroid of the Local Group and which was "hit" by the Earth.

\section{ESTIMATE OF THE SIZE AND MASS OF THE METEOR BODY}

When calibrated to the spectrophotometric standard, the integrated brightness of the meteor estimated from the spectrum shown in Fig. 2 yields apparent V-band magnitudes in the interval $9 \div 12^{m}$ for the observed angular velocities $1 \div 0.1^{\circ} / \mathrm{s}$ of the meteor. We have at our disposal single-site observations of the event, making it impossible to obtain all parameters of its trajectory that are required to correctly and completely solve the inverse problem. We therefore may obtain only very coarse estimates. The estimates of the apparent integrated magnitude of the meteor combined with the velocity distribution along the line of sight imply that we are dealing with the entry into the atmosphere of a rather small body moving at a velocity of $v \geq 300 \mathrm{~km} / \mathrm{s}$. Faint-meteor events usually occur at heights on the order of $100 \mathrm{~km}$ and therefore we set $m$ equal to the absolute magnitude of the meteor. We further assume that $v=300 \mathrm{~km} / \mathrm{s}$.

We now bring the equations of deceleration and mass loss of the meteor body in the Earth's atmosphere to the following form:

$$
D \frac{d v}{d t}=-0.5 C_{x} \rho v^{2}
$$

and

$$
\frac{d D}{d t}=-\frac{\Lambda}{6 Q} \rho v^{3},
$$

where

$$
D=M / S,
$$

$M$ and $S$ are the mass and area of the midsection (the maximum section perpendicular to the direction of motion); $v$, its velocity; $\rho$, the density of the atmosphere; $C_{x}$ and $\Lambda$, the resistance and heat transfer coefficients, respectively; $Q$, the specific energy of destruction, i.e., the energy required to carry unit mass away from the surface of the body [11.

At such heights small bodies interact with the Earth's atmosphere in free-molecule mode. Therefore, according to Kalenichenko [12, we must set $\Lambda=1$ and $C_{x}=2$ in the above equations. Here $Q \leq 10^{11} \mathrm{erg} / \mathrm{g}$, where the upper boundary corresponds, by the order of magnitude, to evaporation of most of the compounds with the allowance for heating from absolute zero and for all the phase transitions [12].

It is easy to verify that under such conditions the body must disintegrate completely before its velocity appreciably decreases. We therefore estimate its size by integrating the equation of mass loss at $v=$ const jointly with the equation of the variation of the density of the atmosphere 


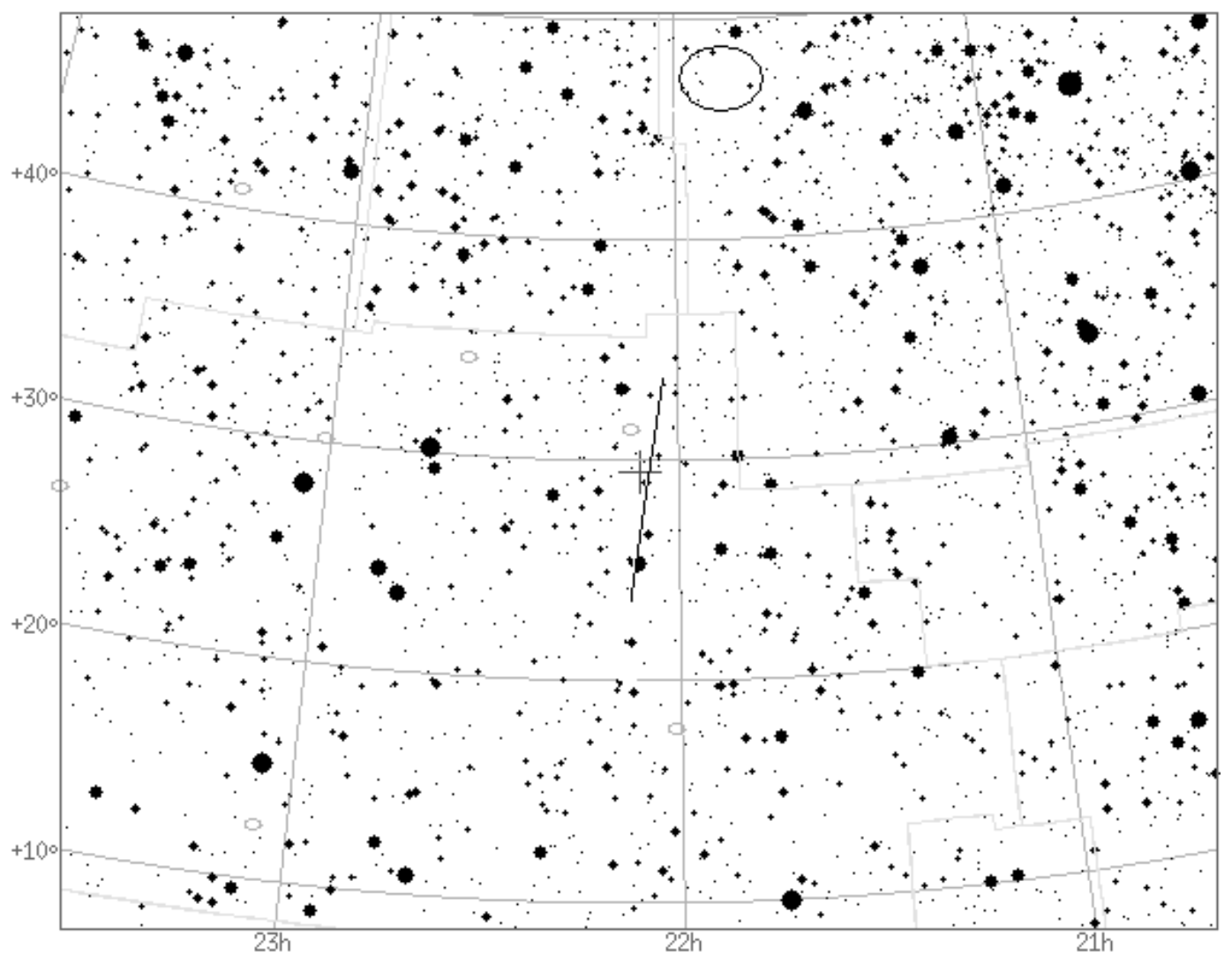

Fig. 4. Portion of the sky map with the ellipse and straight line showing the apex of motion toward the centroid of the Local Group of galaxies and the track of the meteor recorded by the 6-m telescope of the Special Astrophysical Observatory of the Russian Academy of Sciences, respectively.

in the comoving reference frame:

$$
\frac{d \rho}{d t}=\frac{\rho v \cos z}{H}
$$

where $z$ is the angle of entry of the body into the atmosphere counted from the vertical direction (the zenith angle of the radiant of the fireball); $H$, the isothermal atmosphere scale height in the Earth's gravity field. Integrating from infinity $\left(D=D_{0}, \rho=0\right)$ to the disintegration of the body $(D=0, \rho=\rho)$, we obtain:

$D_{0}=\frac{\Lambda H}{6 Q \cos z} \rho v^{2}$,

i.e., a body of size $D_{0}$ can penetrate into the atmosphere down to the layers with density $\rho$. Values of atmosphere density $\rho$ have been calculated for each value of $h$ in accordance with the standard model atmosphere [13. Formula (1) can be used to estimate the lower limit of the characteristic size of the body if we substitute into it the upper limit for $Q$, because, according to Kalenichenko [14,

$$
S=\kappa D^{2} .
$$

In our case we adopt $\kappa=1 \mathrm{~cm}^{6} \mathrm{~g}^{-2}$, which is rather close to the mode of this distribution for a substantially large sample of nondisintegrating fireballs of the Peripheral network [15] computed assuming that the scatter and shapes are the same for both the fireballs and minor bodies. However, in this case it is evident that the value $R_{0}=\sqrt{\kappa D_{0}^{2}}$ characterizes the size of the body. To make estimates convenient, we also assume that $I(\mathrm{erg} / \mathrm{s})$ obeys the well-known (see, e.g., 16] ) dependence on its absolute magnitude $m$ (the magnitude reduced to a distance of $100 \mathrm{~km}$ from the observer),

$$
\lg I=9.72-0.4 m,
$$

and is related to the loss of energy of the incident gas via the midsection of the body by the following formula

$$
I=0.5 C_{H} S \rho v^{3},
$$

where $C_{H}$ is the radiative heat transfer coefficient (see, e.g., [14), implying

$$
S=\frac{2 I}{C_{H} \rho v^{3}}
$$

and characteristic size

$$
R=\sqrt{S}=\sqrt{\frac{2 I}{C_{H} \rho v^{3}}} .
$$

No $C_{H}$ estimates are available for the Earth's atmosphere, not to mention free-molecular flow, at such high hypersonic velocities. We take the liberty to use the $C_{H} \approx 10^{-3}$ value for Prairie Network fireball 40503 
$(v \approx 22 \mathrm{~km} / \mathrm{s})$ at the point of its appearance at a height of about $95 \mathrm{~km}$, where the flow about its body was still free molecular [17.

The Table lists the estimates of the characteristic size of our meteor obtained adopting all the above assumptions.

Estimates of the characteristic size of the meteor body

\begin{tabular}{l|l|l|l}
\hline$h, \mathrm{~km}$ & $R_{0}>, \mathrm{cm}$ & $R, \mathrm{~cm}(m=9)$ & $R, \mathrm{~cm}(m=12)$ \\
\hline 100 & 1 & 0.01 & 0.004 \\
\hline 110 & 0.2 & 0.03 & 0.01 \\
\hline 120 & 0.05 & 0.06 & 0.02 \\
\hline
\end{tabular}

The size of the body can also be estimated in a different way. The meteor moved by at least 3 arcmin during the time while it remained in the field of view of the camera (Fig. 1). This displacement $l$ corresponds to about $100 \mathrm{~m}$, or two orders of magnitude less than $H$, at a height of $100 \mathrm{~km}$. In this case we can neglect the change in $\rho$ over distance $l$ and use the following relation instead of (11):

$D_{0}=\frac{\Lambda}{6 Q} \rho v^{2} l$

This relation yields, under all the above assumptions, the size $R_{0}$ of the meteor body on the order of $0.01 \mathrm{~cm}$ at a height of $100 \mathrm{~km}$ (compare with the values listed in the Table). The result also confirms that our adopted $C_{H}$ value is sufficiently correct. Note that substituting $Q=$ $10^{11} \mathrm{erg} / \mathrm{g}$ into formulas (11) and (2) yields underestimated $R_{0}$ values, and the same is true for the result obtained by substituting the lower boundary for $l$ into formula (2). The actual size of the body may be several times greater than that.

One may expect that the time scale of the disintegration of a particle moving in the Earth's atmosphere at a velocity on the order of $300 \mathrm{~km} / \mathrm{s}$ is less than $0.1 \mathrm{~s}$, and therefore the observed meteor may have an integrated magnitude of more than 9 magnitude. If particles disintegrate at higher altitudes, then their sizes must be greater all other conditions being the same. Therefore the characteristic size listed in the table is a lower estimate. We adopt a burnout height of $h>100 \mathrm{~km}$ to infer a size estimate of $R>10^{-2} \mathrm{~cm}$. If the observed meteor particle is a chondrite (with a density of about $3 \mathrm{~g} / \mathrm{cm}^{3}$ ), then the mass of the particle can be estimated at $M>7 \cdot 10^{-6} \mathrm{~g}$.

Note that our estimate, which we obtained under rather coarse assumptions, agrees with numerical computations of the expected parameters (magnitudes and masses) of interstellar meteors moving with velocities as high as $500 \mathrm{~km} / \mathrm{s} 18$. Hill et al. 18 showed that meteors with the velocities of $300 \mathrm{~km} / \mathrm{s}$ and masses on the order of $10^{-5} \mathrm{~g}$ must be observed as about $9^{m}$ objects disintegrating at the heights of $110-120 \mathrm{~km}$.

It is interesting that our estimate coincides with the size of chondrules in carbonaceous chondrites (see, e.g.,
[19)], which are usually smaller than $0.5 \mathrm{~mm}$ and constrained to a rather narrow interval. Many chondrules have spherical or spheroidal shapes and appear to have formed as a result of fast crystallization and hardening of melt drops. The distribution of carbon isotopes in carbonaceous chondrites differs widely from that of terrestrial and lunar rocks and is suggestive of extrasolar and (or) presolar (pregalactic) material.

\section{ESTIMATE OF THE NUMBER OF HIGH-VELOCITY METEORS}

By itself, the fact of observing a high-velocity meteor provides no information about the number of such particles, and the coincidence of the direction of its trajectory with the direction toward the apex of motion toward the centroid of the Local Group of galaxies does not prove the extragalactic nature of the meteor. To estimate the number of high-velocity meteors and search for the position of their radiant near the apex, we observed in October-November, 2006 faint sporadic meteors with FAVOR wide-angle CCD camera developed at the Special Astrophysical Observatory of the Russian Academy of Sciences and Federal Research Institute of Semiconductor Devices [20]. The camera has a $16 \times 22^{\circ}$ field of view; pixel size of 1.5 acrmin; single-frame exposure of $0.12 \mathrm{~s}$; limiting $\mathrm{V}$-band magnitude of 10.5 , and a signal-to-noise ratio of 5 .

We conducted observations in six fields located around the apex for a total time of 34.5 hours during six observing nights to take about $10^{6}$ frames from which we selected about 2000 ones with recorded meteors. After astrometric calibration we determined the coordinates of the beginning and end of the meteor tracks. The track morphology and repeated track images (for meteors moving with low angular velocities) allowed us to determine the direction of the meteor motion on the sky. We identified a total of 246 meteors with a mean magnitude of $\mathrm{V} \approx 7^{m}$ in the field studied. The magnitudes of the faintest of these meteors lied in the $9-9.5^{m}$ interval. The median angular velocity of the meteors found is equal to $0.8^{\circ} / \mathrm{s}$. We show their tracks as gray dashes in Fig. 5 .

To verify whether the field of recorded meteors had a radiant, we tested the coincidence of the direction of the radius vector pointing from the given radiant point to the start of the track with the direction of the apparent trajectory of the meteor. We varied the position of the radiant within $\pm 10^{\circ}$ in both coordinates about the apex position. The size of the error box for the track direction is about 3 degrees and it is determined by the accuracy of astrometric calibration (about $20^{\prime \prime}$ ). The left-hand panel in Fig. 6 shows the distribution of the number of meteors that obey the condition of emerging from the radiant and the observed density of the occurrence frequency of such meteors in the sky plane. It is evident from the figure that about 12 meteors out of 246 obey the condition of motion from the radiant, and the position of the radiant can be confidently determined from the maximum of the distribu- 


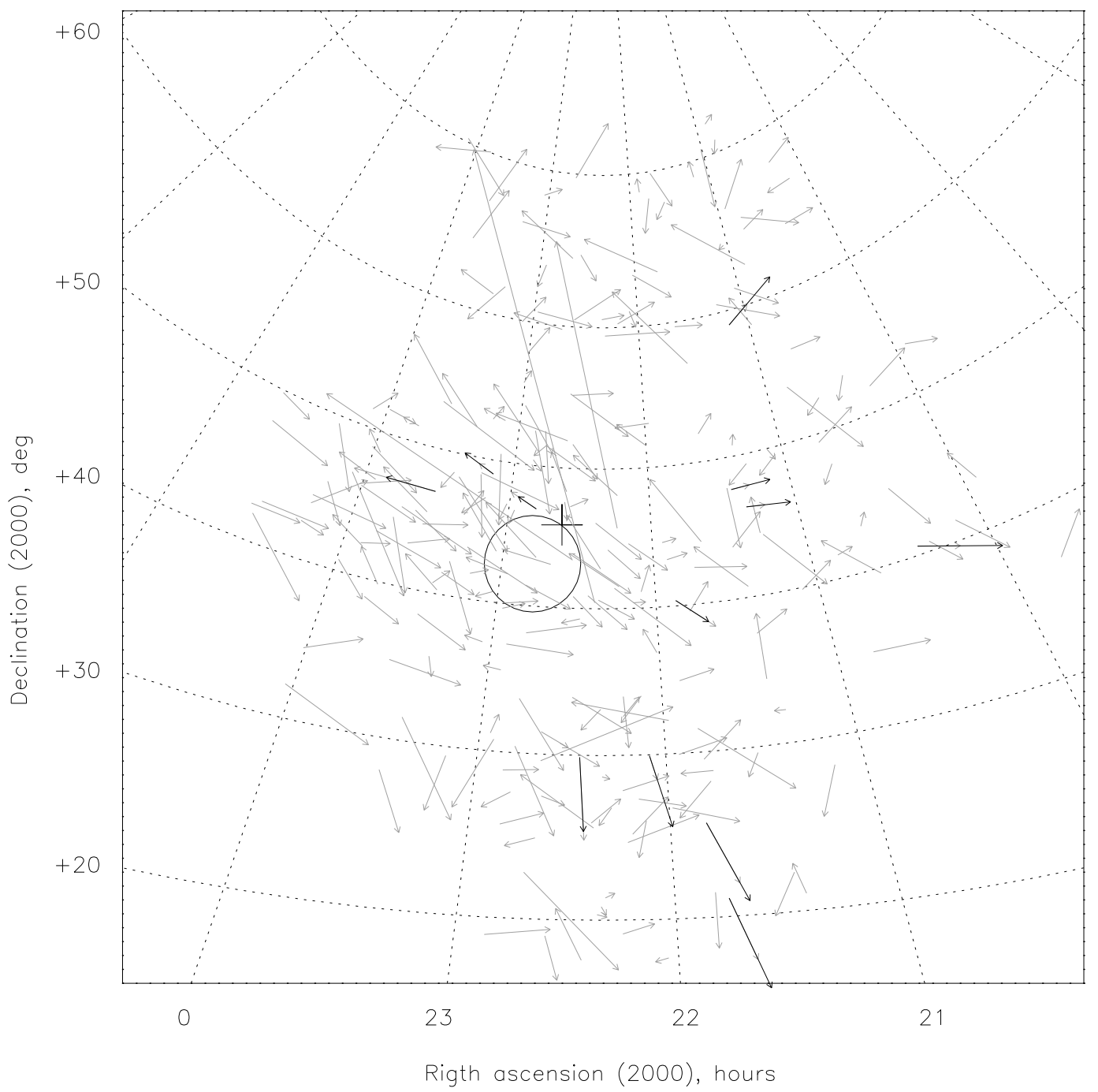

Fig. 5. Tracks of meteors recorded with FAVOR wide-angle camera. The black arrows indicate the meteors emerging from the radiant, which we marked by the cross, and the circle shows the position of the apex of motion toward the centroid of the Local Group of galaxies.

tion function of the occurrence frequency in the sky plane. For a $3^{\circ}$ error box the expected number of meteors should be equal to about three in the case of a random distribution of coordinates and orientations of tracks. In our field the distribution of track coordinates is not uniform and to test the statistical significance of the result obtained, we numerically simulated tracks with the coordinates coinciding with those of the observed tracks and accidentally distributed directions (with uniformly distributed angles). Figure 6 shows the result of our estimate of the number of meteors satisfying the condition of motion from the radiant for the case of accidental distribution of track directions. It follows from this figure that the number of such meteors is equal to about five for a random model, but there is no distinguished point in the distribution of the occurrence frequency on the sky plane. Judging by the form of the distributions of observed track directions for the random-distribution model for the observed track directions, our radiant located near the apex of the motion toward the centroid of the Local Group of galaxies is not accidental at a confidence level of $¿ 96 \%$. In Fig. 5 the black arrows, circle, and cross show the meteor tracks satisfying the condition of motion from the radiant, the position of the apex corrected for the motion of the Earth, and the inferred position of the radiant, respectively.

Given the distance between the track and the radiant and the track length, one can estimate the geocentric velocity of the meteors. We obtain a velocity estimate of $282 \pm 53 \mathrm{~km} / \mathrm{s}$ for a $110 \mathrm{~km}$ intrusion height into the Earth's atmosphere and an average magnitude of 8.2. Note that the estimates of the velocity and brightness of meteors so obtained agree with spectroscopic data.

\section{ESTIMATE OF THE SPACE DENSITY OF INTERGALACTIC DUST}

Observations of the flares of high-velocity particles in the upper layers of the atmosphere allow us to estimate the 

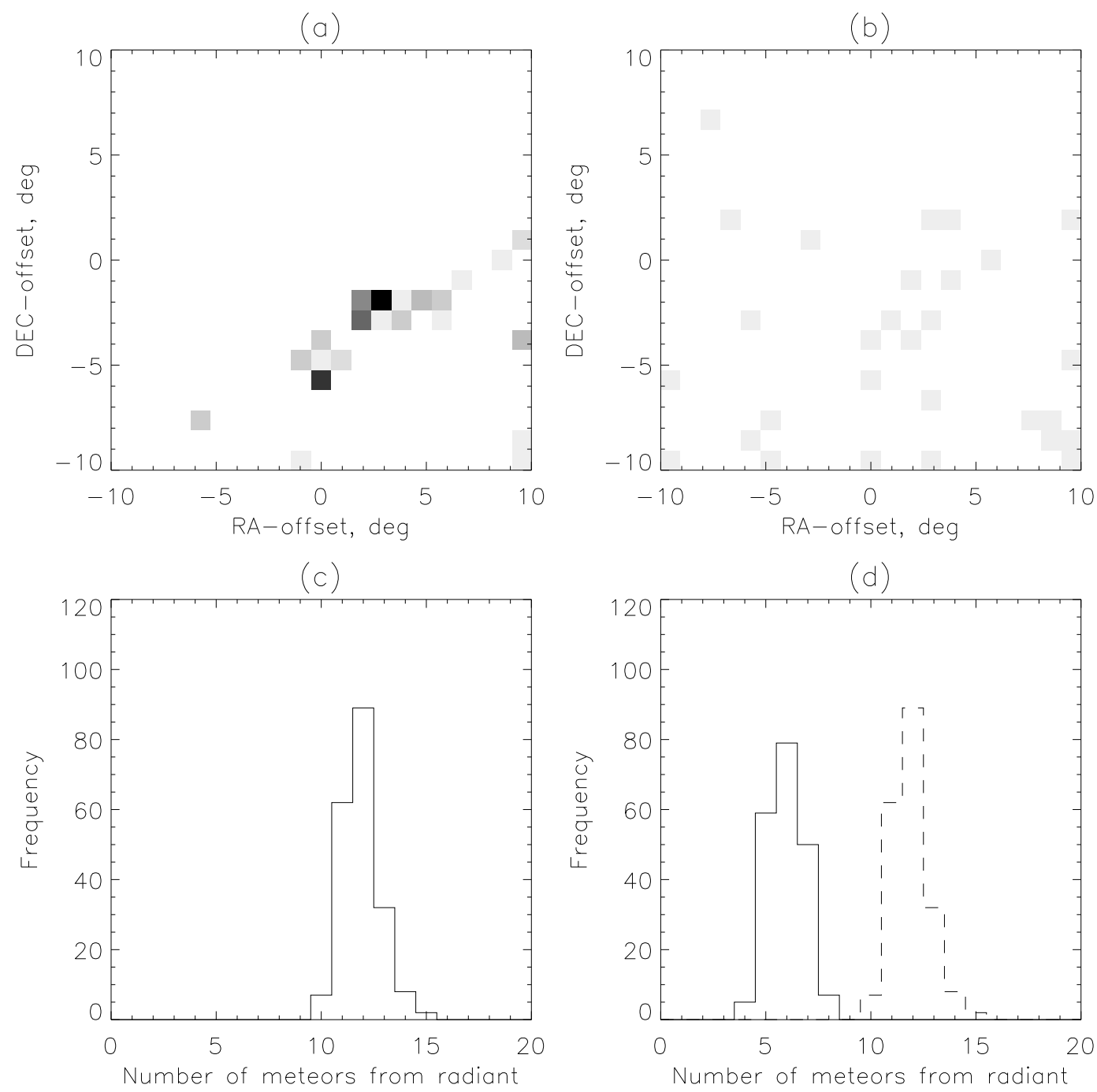

Fig. 6. Distribution of the occurrence frequency of meteor tracks satisfying the condition of emergence from the radiant with the given coordinates. (a) The observed two-dimensional distribution of the occurrence frequency of meteors for different coordinates of the radiant with respect to the center with RA $22^{h} 20^{m}$ and DEC $+48^{\circ}$, (c) the distribution of the observed number of meteors satisfying the condition of emergence from the radiant. (b) and (d) Same as figures (a) and (c), but for the model of randomly distributed track orientations. The dashed line shows the observed distribution of the number of tracks to be compared with the model shown in the figure (d).

space density $n_{d}$ of such particles. Given that the velocity $V_{d}$ of fast dust particles exceeds the velocity of the Earth by one order of magnitude, space density $n_{d}$ can be estimated as

$$
n_{d}=\left(S_{0} V_{d} t\right)^{-1}
$$

where $S_{0}$ is the survey area during the search for events (flares), and $t$ is the average time interval between observed events. In our case for the patrol field of view with the size of about $40^{\circ}$ the survey area at the altitude of $\sim 110 \mathrm{~km}$ and a mean zenith distance of $z \approx 40^{\circ}$ is equal to $S_{0} \approx 10^{14} \mathrm{~cm}^{2}$. The data reported above allow us to adopt $\mathrm{t} \approx 3^{h} \approx 10^{4} \mathrm{~s}$ as the average time interval between events in this field. For a velocity of $V_{d} \approx 300 \mathrm{~km} / \mathrm{s}$ we obtain $n_{d}=3.6 \cdot 10^{-26} \mathrm{~cm}^{-3}$. For a typ- ical dust-grain mass of $M_{d} \geq 0.7 \cdot 10^{-5} \mathrm{~g}$, we find the average density of intergalactic particles in the Earth's vicinity $\rho \geq 2.5 \cdot 10^{-31} \mathrm{~g} / \mathrm{cm}^{3}$, i.e., about $2.5 \%$ of the critical density.

Let us assume that extragalactic dust with the above density is uniformly distributed over the entire volume of the Local Group. According to Karachentsev [21, Local Group of galaxies can be characterized by its "zero velocity sphere" radius $R_{L G}=(1 \pm 0.1) \mathrm{Mpc}$ and total mass $M_{L G}=(1.3 \pm 0.3) \cdot 10^{12} M_{\odot}$. The total mass of dust in this volume is equal to $1.6 \cdot 10^{10} M_{\odot}$, or about $1 \%$ of the total mass of the group. It does not appear unusual, because such a mass has no effect on the dynamics of the Local Group of galaxies.

On the other hand, the intergalactic dust medium may show up in integrated or selective absorption. Let us as- 
sume that this medium with density $n_{d}$ and characteristic dust-grain radius of $R_{d} \sim 10^{-2} \mathrm{~cm}$ uniformly fills the Universe out to the horizon $\mathrm{R}_{c}=\mathrm{c} / \mathrm{H}_{0}$, where $\mathrm{c}$ is the speed of light and $\mathrm{H}_{0}$, the Hubble constant $(72 \mathrm{~km} / \mathrm{s} / \mathrm{Mpc})$. In this case the optical depth of the dusty Universe is

$$
\tau=\pi R_{d}^{2} n_{d} c / H_{0},
$$

if we neglect various evolutionary effects. The above estimate of $n_{d}=3.6 \cdot 10^{-26} \mathrm{~cm}^{-3}$ implies an optical depth of the Universe of only $\tau \approx 0.15$, which would have no apparent effect on the spectra of distant galaxies and quasars. In reality, one may expect that intergalactic dust should concentrate in groups and clusters of galaxies. According to Karachentsev and Lipovetsky [22], the optical depth of clusters of galaxies does not exceed $\tau \approx 0.2$, implying no conflicts with observational data.

Of course, the following important questions remain open:

(1) How have metal-rich dust particles come to the intergalactic space? In principle, various mechanisms may explain this - supernova explosions, tidal interactions of galaxies, radiation pressure, condensation in molecular clouds (chondrules?), etc.

(2) Why are the sizes of intergalactic particles two orders smaller (and their masses are six orders of magnitude greater) than common interstellar dust particles in our Galaxy?

(3) If intergalactic dust associates with galaxies in the form of dust aureoles, is it possible now to find such formations (IR observations onboard Spitzer)? As is well known, the aureoles of ionized gas around nearby spiral galaxies are already observed [23].

(4) If nonuniform intergalactic dust medium with the above parameters does exist, would it show up as irregularities on cosmic microwave radiation (WMAP etc.)?

We expect to answer these questions in the nearest future.

Acknowledgements. We are grateful to E. Katkova for performing observations with FAVOR camera, S. Karpov for preliminary reduction of real-time data, and to R.Z. Sagdeev and A.M. Fridman for fruitful discussions.

\section{References}

[1] M. Hapgood, P. Rothwell, O. Royvik, MNRAS 201, 569, (1982).

[2] J. Borovicka, R. Stork, J. Bocek, Meteorics and Planetary Science, 34 987, (1999).

[3] J. Stauffer, H. Spinrad, PASP 90, 222, (1978).

[4] V.L. Afanasiev, A.V. Moiseev, V. L. 2005, Astronomy Letters, 31, 193; (astro-ph/0502095)

[5] J. Borovicka, O. P. Popova, A. P. Golub, et al., A\&A 33, 591, (1998).

[6] J. M. Trigo-Rodriguez, J. Llorca, J. Borovicka, J. Fabrecant, Meteorits \& Planetary Science 38, 1283, (2003).

[7] V. A. Kamenshchikov, Yu. A. Plastinin, V. M. Nikolaev, L. A. Novitskii, Radiatsionnye Svoistva Gazov pri Vysokikh Temperaturakh (Radiation Properties of Gases at Hight Temperatures), ( Mashinostroenie, Moscow, 1971) (in Russian).
[8] D. P. Galligan, W. J. Baggaley, MNRAS 359, 551, (2005). [9] A. D. Taylor, W. J. Baggaley, R. G. T. Bennet, D. I. Steel, Planetary and Space Science 42, 135, (1994).

[10] I. D. Karachentsev, D. I. Makarov, AJ, 111, 794, (1996).

[11] V. V. Kalenichenko, Kinematica Fiz. Nebesnykh Tel 8, 69, (1992).

[12] V. V. Kalenichenko, Astron. Vetsnik. 14, 86, (1982).

[13] CIRA 1972, Cospar International Reference Atmosphere (Berlin, Akademie-Verlag, 1972), 450 pp.

[14] V. V. Kalenichenko, Kinematica Fiz. Nebesnykh Tel 8, 61, (1992).

[15] V. V. Kalenichenko, Kinematica Fiz. Nebesnykh Tel 9, 52, (1993).

[16] V. A. Bronshten, Fizika Meteornykh Yavlenii (Physics of Meteor Phenomena), (Nauka, Moscow, 1981), 416 (in Russian).

[17] V. V. Kalenichenko, Kinematica Fiz. Nebesnykh Tel 20, 157, (2004).

[18] K. A. Hill, L. A. Rogers, R. L. Hawkes, A\&A 444, 615, (2005).

[19] R. T. Dodd, Meteority. Petrologiya i Geokhimiya, (Mir, Moscow, 1986), 384 (in Russian). Translated from R. T. Dodd. Meteorites - a Petrologic-Chemical Synthesis. Chemistry University Press, 1981. 368 pp

[20] S. Karpov, G. Beskin, A. Biryukov, et al., I., IL NUOVO CIMENTO 28, 747, (2005).

[21] I.D. Karachentsev, AJ, 129, 17, (2005)

[22] I. D. Karachentsev and V. A. Lipovetsky, Astron. Zh. 45, 1148, (1968).

[23] F. Fraternali, J. Binney, T. Oosterloo,and R. Sancisi, New Astronomy Review 51, 95, (2007). 